\title{
Komplikationsreicher Verlauf nach Schultergelenksinjektionen
}

\section{Hans-Jörg Oestern}

\section{Eine intraartikuläre Injektion und ihre Folgen}

Die Zahl der Gelenkinfekte nach Injektionen ist vergleichsweise gering. Tritt eine Infektion ein, ist sie immer mit einem längerfristigen Krankenhausaufenthalt verbunden, im Extremfall sind auch tödliche Komplikationen beschrieben [4].

\section{Kasuistik}

Anamnese

Es handelt sich um eine 65-jährige Patientin, die angab, dass sie seit etwa 14 Tagen Schmerzen im Bereich des rechten Schultergelenks gehabt habe (Abb.1). Es seien mehrere Injektionen in das Gelenk verabreicht worden.

Die Beschwerden seien nicht besser geworden, sondern hätten sich im Gegenteil deutlich verstärkt.

\section{Aufnahmebefund}

Bei der stationären Aufnahme zeigte sich eine diffuse Rötung im Bereich der rechten Schulterregion, vor allem im Bereich des M.trapezius. Die Beweglichkeit des Gelenks war schmerzhaft aufgehoben. Über dem Processus coracoideus fand sich eine fluktuierende Schwellung.

Bei der Sonografie des Gelenks konnte eine intraartikuläre Ergussbildung nachgewiesen werden.

Zu diesem Zeitpunkt wurden auch bereits Schmerzen in beiden Oberschenkeln angegeben. Im Bereich des linken Fußrückens zeigte sich eine entzündli-

OP-JOURNAL 2008; 24: 200-201

(c) Georg Thieme Verlag KG Stuttgart • New York DOI 10.1055/s-2008-1039151 che Schwellung, die 2. Zehe wies eine feuchte Gangrän auf.

Laborchemisch bestand eine hochgradige Erhöhung der CRP auf 49,6 mg/dl (Normalwert 0,5 mg/dl) sowie eine Leukozytose von 18900 Zellen $/ \mathrm{mm}^{3}$.

\section{Therapie am Aufnahmetag}

Zwei Stunden nach Aufnahme erfolgte die operative Revision des Schultergelenks, wobei subdeltoidal und im Gelenk der Infekt nachweisbar war. Außerdem bestand eine Ruptur der Rotatorenmanschette. Es erfolgte ein ausgedehntes Débridement mit Drainage des Subakromialraums und des Gelenks.

In der gleichen Sitzung wurde die gangränöse 2. Zehe amputiert.

Trotz dieser Maßnahmen entwickelte sich wenige Stunden später ein septisches Krankheitsbild mit Kreislaufversagen und Ateminsuffizienz.

\section{Weitere Therapie}

Es erfolgte die Verlegung auf die Intensivstation. Hier konnte der Kreislauf nur durch Katecholamin-Gaben gestützt werden. Aufgrund der respiratorischen Insuffizienz war eine kontrollierte Beatmung erforderlich. Als Ursache für die Sepsis wurde Staphylococcus aureus in den Abstrichen des Schultergelenks nachgewiesen. In der Folgezeit erfolgten 4 weitere Revisionen im Schulterbereich.

Dennoch verschlechterte sich der Allgemeinzustand zunächst weiter. Daneben entwickelte sich noch eine heparininduzierte Thrombozytopenie. Im Bereich des Unterschenkels kam es bei hochgradiger AVK zu einer Ischämie, die eine Unterschenkelamputation erforderlich machte. Die Patientin entwickelte dennoch eine hochgradige respiratorische Insuffizienz und musste mit

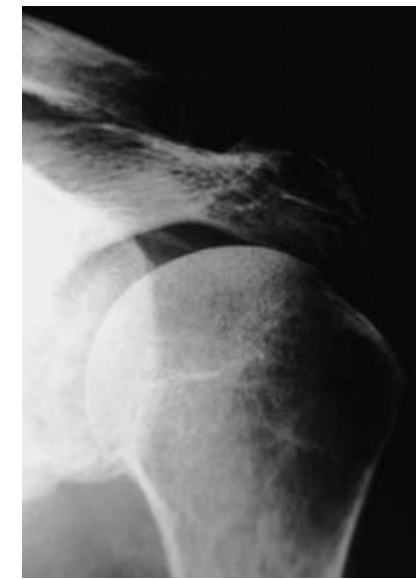

Abb. 1 Röntgenbild des Schultergelenks mit ImpingementSyndrom.

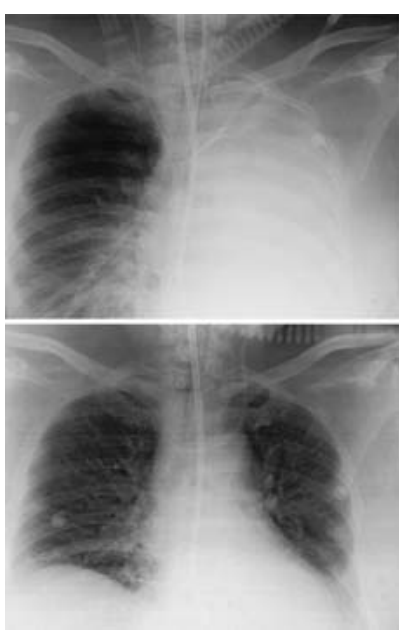

Abb. 2 Schwere respiratorische Insuffizienz mit Verschattung der linken Lunge. Ergussbildung. Während dieser Zeit wurde die Patientin mit 100\% Sauerstoff beatmet.

$100 \%$ Sauerstoff beatmet werden (Abb. 2). Die arteriellen Blutgase verschlechterten sich weiter. Neben Drehbettlagerung erfolgte Hämofiltration, worunter sich die Patientin langsam stabilisierte. 

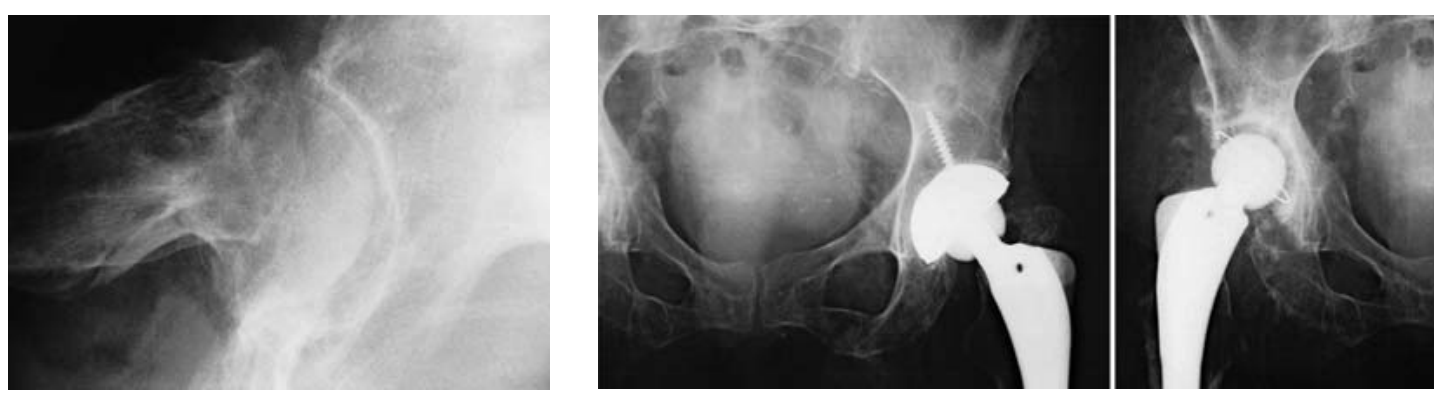

Abb. 3 Mediale Schenkelhalsfraktur.

Nach mehrwöchiger intensivmedizinischer Behandlung konnte die Patientin auf die periphere Station verlegt werden.

Nach 2 Wochen kam es jedoch zu einem 2. septischen Schub, dessen Ausgangsherd zunächst nicht identifiziert werden konnte.

Nachdem computertomografisch flüssigkeitsgefüllte Hohlräume in der Milz nachgewiesen wurden, erfolgte unter dem Verdacht von Milzabszessen eine Splenektomie.

Histologisch ließen sich infarktgeschädigte Areale und kleinere Abszedierungen nachweisen.

Die Patientin wurde nochmals 6 Wochen beatmet, bis sie schließlich nach 122-tägiger intensivmedizinischer Behandlung und 94-tägiger Beatmung entlassen werden konnte.

Im weiteren Verlauf erlitt die Patientin im Zeitraum von einem Jahr Schenkelhalsfrakturen rechts und links, die die Implantation jeweils einer TEP notwendig machten (Abb. 3 und 4).

Inzwischen ist die Patientin mit einer Unterschenkelprothese gut gehfähig und kann ihren Haushalt alleine versorgen.

\section{Diskussion}

Prädisponierende Faktoren für eine septische Arthritis stellen Immundefekte oder Immunsupression, z. B. nach Transplantation, HIV-Infektionen, sowie vorbestehende Malignome, systemische Steroid-Therapie sowie Injektionen und Punktionen und vorausgegangene operative Eingriffe dar. Unter der großen Zahl intraartikuläer Punktionen und Injektionen ist die Infektionsrate relativ gering. Anders [1] gab im Rahmen einer
Sammelstudie bei über 650000 intraartikulären Injektionen und Gelenkpunktionen die Inzidenz eines Gelenkempyems mit 0,034\%。 an. Werden zusätzlich Kortikoide injiziert, ist die Infektionsrate höher [2,3]. Die Symptomatik der bakteriellen Infektion ist geprägt vom Gelenkschmerz, der in der Regel 2 bis 3 Tage nach einer Injektion einsetzt und besonders nachts auftritt. Jeder chronische Schmerzzustand, der sich verschlimmert und mit Injektionen angegangen wurde, ist verdächtig auf einen Gelenkinfekt und muss so lange untersucht werden, bis eine eindeutige Diagnose feststeht. In den laborchemischen Entzündungsparametern sind z. B. C-reaktives Protein und die Leukozytenzahlen die wichtigsten Parameter. Unter den Erregern stehen die grampositiven Bakterien im Vordergrund. Ein Keimnachweis ist jedoch nicht immer zu erbringen und die Rate negativer Abstriche schwankt in der Literatur zwischen 6 und 33\%. Für die Prognose entscheidend ist die frühzeitige Diagnosestellung und daraus folgernd die rasche Therapie. Innerhalb von Stunden verursachen die intraartikulären Bakterien eine humorale und zelluläre Entzündungsreaktion der Synovialmembran mit leukozytärer Infiltration und begleitendem Gelenkerguss. Schon ab dem 3. Tag zeigt die Synovialmembran reaktive Veränderungen, die eine Restitutio ad integrum unmöglich machen. In der Diagnostik des akuten Infekts sind deshalb auch zeitaufwendige Untersuchungsverfahren wie Szintigrafie weniger hilfreich, ganz im Gegensatz zum chronischen Infekt. Die Therapie beim akuten Schultergelenksinfekt ist die sequenzielle Arthroskopie. Bei weiter fortgeschrittenen Infekten, wie im vorliegenden Fall, mit flächenhafter Rötung ist ein offenes Vorgehen anzustreben. Wichtig ist das mehrmalige Débridement, welches im 48-Stunden-Rhythmus bis zur Keimfreiheit durchgeführt werden muss.
Abb. 4 Totalendoprothesen beidseits.
Fazit

Aufgrund der pathophysiologischen Veränderungen beim Gelenkinfekt kommt der schnellen Diagnostik eine entscheidende Bedeutung zu. Anamnese, klinischer Befund, C-reaktives Protein und Leukozyten sowie Sonografie und Gelenkpunktion erhärten die Diagnose. Eine Gramfärbung vom Punktat erlaubt den Nachweis von Erregern. Initial wird mit der Gabe eines staphylokokkenwirksamen Antibiotikums begonnen, bis die Resistenzbestimmung vorliegt. Die arthroskopisch assistierte Spülung ist das Standardverfahren beim Gelenkinfekt. In Einzelfällen, wie in diesem Fall, kann aufgrund der flächenhaften Ausdehnung des Infektgeschehens eine offene Vorgehensweise indiziert sein. Rezidivierende arthroskopische Spülungen bzw. Redébridements sind bis zum negativen Keimnachweis unumgänglich.

\section{Literatur}

${ }^{1}$ Anders G. Gelenkpunktionen und intraartikuläre Injektionen in ambulanten orthopädischen Einrichtungen. Beitr Orthop Traumatol 1984; 31: 419-425

2 Armstrong RW, Bolding F. Septic arthritis after arthroscopy: the contributing roles of intraarticular steroids and environmental factors. Am J Infect Control 1994; 22: 16-18

${ }^{3}$ Burdge DR, Reid GD, Reeve CE et al.. Septic arthritis due to dual infection with Mycoplasma hominis and Ureaplasma urealyticum. Rheumatology 1988; 15: 366-368

${ }^{4}$ Esenwein SA, Ambacher T, Kollig E, KutschaLissberg F, Hopf F, Muhr G. Septische Arthritiden des Schultergelenks nach intraarticulärer Injektionstherapie. Unfallchirurg 2002; 105: 932-938

\section{Prof. Dr. med. Hans-Jörg Oestern} Chefarzt

Klinik für Unfallchirurgie, Orthopädie und Neurotraumatologie

Allgemeines Krankenhaus Celle

Siemensplatz 4

29223 Celle

E-Mail: hans-joerg-oestern@ akh-celle.de 